\title{
Revista de Estudios Sociales
}

10 | Octubre 2001

La Ciudad y las Ciencias Sociales (I)

\section{The Global City. New York, London, Tokyo}

Saskia Sassen, Princeton-New Jersey, Princeton University Press, 1991, $397 \mathrm{p}$.

Luis Mauricio Cuervo

\section{OpenEdition}

Journals

Edición electrónica

URL: https://journals.openedition.org/revestudsoc/27961

ISSN: $1900-5180$

Editor

Universidad de los Andes

Edición impresa

Fecha de publicación: 1 octubre 2001

ISSN: 0123-885X

\section{Referencia electrónica}

Luis Mauricio Cuervo, «The Global City. New York, London, Tokyo», Revista de Estudios Sociales [En línea], 10 | Octubre 2001, Publicado el 22 mayo 2019, consultado el 04 mayo 2021. URL: http:// journals.openedition.org/revestudsoc/27961

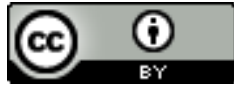

Los contenidos de la Revista de Estudios Sociales están editados bajo la licencia Creative Commons Attribution 4.0 International. 


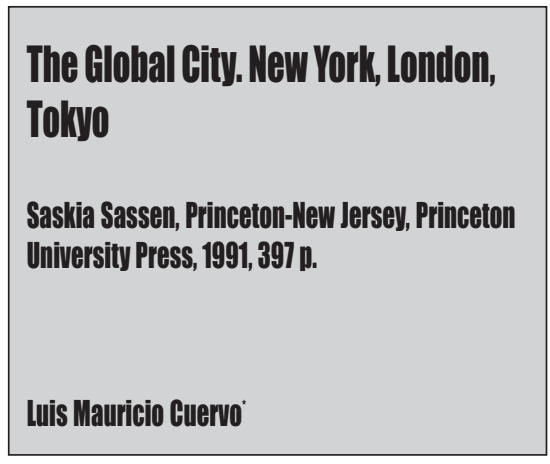

Comentario general

Es un trabajo de excelente calidad que con razón ha marcado un hito en la investigación urbana mundial durante la década de los años 1990. Aborda un tema abundantemente estudiado y discutido por la literatura especializada hasta ese momento, 1991, con algunas características que probablemente explican su originalidad y su impacto. En primer lugar cabe destacar el detalle y la minucia con la que son tratadas las hipótesis y elementos centrales de la argumentación: hace un esfuerzo destacable y significativo por precisar la magnitud y las características de cada uno de los fenómenos cruciales detrás de cada una de las hipótesis. Este esfuerzo se hace tanto en el campo de la revisión teórica de los antecedentes, de las formas posibles de aproximación al problema, como en el de la caracterización empírica.

En segundo lugar, debe señalarse el cuidado lógico con el que es abordado cada componente de la argumentación. El trabajo es conciente de la dificultad de definir la peculiaridad de la globalización como época particular del desarrollo capitalista mundial y, en

* Economista de la Universidad de los Andes y Doctor en Urbanismo del Instituto de Urbanismo de Paris, Universidad de Paris XII. Profesor Titular de la Universidad de los Andes. Director del Centro Interdisciplinario de Estudios Regionales (CIDER) desde 1998 concordancia, hace el esfuerzo por poner de presente en qué consiste la particularidad de la época en cuestión, tanto en lo financiero, como en los servicios, las escalas geográficas del análisis, etc.

Finalmente podría subrayarse el cuidadoso manejo del siempre difícil arte del análisis comparativo que en este caso involucra tres países distintos y tres áreas metropolitanas específicas como son Nueva York, Londres y Tokio. Aunque tiende a señalar aquellos aspectos en donde hay evoluciones convergentes, hace un esfuerzo significativo por poner en evidencia las especificidades de cada contexto.

\section{Reflexiones}

El trabajo, a pesar de su reconocimiento mundial y su influencia en la investigación urbana contemporánea, no ha sido suficientemente explotado para nutrir algunos debates estratégicos de teoría y política urbanas en el mundo contemporáneo. A continuación algunas de las reflexiones suscitadas a este nivel.

Un aspecto central se ocupa del tratamiento de la relación entre cambio económico y transformaciones socioespaciales. El trabajo de Sassen no rompe el molde convencional de tratamiento de esta relación, es decir, de pensar el cambio económico como la variable independiente y determinante de las transformaciones socioespaciales. No obstante las posibles críticas a esta forma de aproximación, la manera en que Sassen la desarrolla le permite superar algunas limitaciones de quienes adoptan esta visión de forma poco fundamentada y altamente especulativa. Como dijimos en el parágrafo anterior, Sassen es cuidadosa y rigurosa en el tratamiento de esta relación por varias razones.
Primero, se esfuerza por demostrar teórica y empíricamente sus axiomas centrales. Su punto de partida central es el papel protagónico de lo financiero y de la producción de servicios como características peculiares de la fase actual de la mundialización del capital. Sin darlo por conocido, se esfuerza por mostrar su importancia en términos de magnitudes y modalidades. Así, logra sustentar la emergencia de nuevas realidades económicas propias de la fase analizada, y, adicionalmente, ilustrar su peso y proyección. Se trata, por tanto, de nuevas formas de organización de la actividad económica, con un rol central en la modificación de las condiciones generales de acumulación, trabajo y reproducción social. El gran error cometido por aproximaciones que aceptan la predominancia de lo económico sobre lo socio-espacial es su comodidad de dar por sentados los cambios y no esforzarse por comprender ni sus magnitudes, ni sus modalidades.

Segundo, tampoco da por sentado el rol protagónico de las ciudades globales en el desarrollo de estos nuevos parámetros de la economía, sino que se esfuerza por demostrarlo, poniendo en evidencia la importancia absoluta, relativa y la especificidad cualitativa de estas actividades en estas ciudades. Se trata de cambios de la economía mundial en general con expresiones muy específicas en estas ciudades, que asumen papeles muy precisos y significativos, adecuadamente ilustrados por Sassen.

Por tanto, Sassen muestra la magnitud de los cambios, la novedad de las modalidades de desarrollo adoptadas por las industrias financiera y de servicios, y la centralidad del papel jugado por las ciudades globales en este proceso. Desde este punto de vista construye las bases que la autorizan a 
proponer una relación de dependencia y causalidad desde lo económico hacia lo socio-espacial. La argumentación, su fundamento y desarrollo son suficientemente sólidos como para hacer de sus proposiciones juicios bien construidos y aceptables en su calidad de afirmaciones sólidas. No obstante, esta argumentación, como cualquier otra de carácter científico, se mueve dentro de parámetros que acotan su validez y restringen sus posibles campos de aplicación. Adicionalmente, algunos componentes puntuales de la argumentación dejan dudas e interrogantes y llaman al debate y a la discusión.

\section{Los parámetros de la argumentación y algunos de sus vacíos}

Estos parámetros serán examinados en relación a tres grandes niveles: el tiempo, las escalas, y la estructura de concentración.

\section{i) El tiempo}

Un primer y muy importante parámetro de la argumentación es temporal. El trabajo de Sassen da cuenta de la evolución de la economía mundial y del papel de las ciudades globales en la década de los años 1980. Es sabido que la globalización inicia en los setenta y transita por tres grandes ciclos que han tendido a coincidir con los decenios: setenta, ochenta y noventa. Cada uno con características propias en muchos sentidos pero muy especialmente en uno central para el trabajo de Sassen: las formas específicas de circulación internacional de los recursos de ahorro e inversión. En este sentido, es importante no pasar por alto que el sentido de ciertas tendencias, especialmente las relacionadas con el papel ascendente de la economía japonesa y con su carácter de exportador neto de capital, se transformaron radicalmente durante los años 1990, tanto por la prolongada crisis japonesa, como por la recuperación de la economía norteamericana y su tendencia de crecimiento sostenido. Estas precisiones seguramente no invalidan los aspectos centrales de la argumentación de Sassen, aunque sí modifican algunas de sus expresiones urbanas y geográficas.

Sassen no desconoce el papel de estos parámetros y se pregunta explícitamente por la durabilidad del modo de crecimiento, poniéndola en cuestión. Por tanto, los errores de proyección lineal o trasposición mecánica que se cometan en este sentido son más atribuibles a limitaciones de los lectores del trabajo que al trabajo en sí mismo. Hay un aspecto central de los parámetros temporales de la reflexión sobre las ciudades globales, no mencionada por Sassen pero de particular interés para la teoría y la política urbana. Es evidente que las bonanzas financiera y terciaria llegan a ciudades como Nueva York y Londres (no es el caso de Tokio) en medio de unas de sus más intensas y profundas crisis económicas, sociales y fiscales. Este renacimiento urbano es algo poco previsible y muchas de las proyecciones del momento apostaban a una prolongación de esta crisis urbana y al surgimiento de nuevos polos. De hecho, muchas de las ciudades industriales que las acompañaron en la decadencia, no lograron, y probablemente algunas de ellas no lo han conseguido todavía, detener el descenso
(Detroit, Chicago en los EE.UU. y las ciudades industriales del norte en la Gran Bretaña).

Por tanto, la posición de estas ciudades en su rol global aparece en un momento de crisis y contribuye a una acelerada transformación de estas ciudades y a una suerte de renacimiento bajo parámetros totalmente diferentes. Hay un proceso de acelerado y profundo cambio urbano que no es analizado por Sassen porque no hace parte de sus preocupaciones centrales. No obstante, es bueno no pasarlo por alto porque constituye una interrogante, una incógnita central en materia de política urbana: ¿cuál fue el papel jugado por los distintos procesos, los diferentes actores $y$, más precisamente, los esfuerzos colectivos voluntarios por responder a esta crisis? ¿qué se puede aprender para otros casos? Adicionalmente, cabe preguntarse acerca de si los efectos de polarización social se prolongaron y ampliaron durante la década de los noventa, o se transformaron. Es sabido que el último decenio del siglo XX fue favorable en materia de estabilidad y crecimiento, especialmente para los países desarrollados y que, probablemente, algunos de los negativos signos sociales de la globalización hayan podido transformarse durante esta última década.

Por otra parte, a pesar de las especificidades temporales y geográficas del argumento desarrollado por Sassen, es sorprendente que algunos de los procesos descritos por ella para las ciudades globales se reprodujeron en algunas de las grandes ciudades latinoamericanas durante los noventa. Estos procesos, aunque no 
se explican por el hecho de que estas ciudades jueguen un rol central en la economía financiera y terciaria mundial, si hacen parte del mismo proceso puesto que se relacionan con el papel jugado por América Latina como receptor masivo del ahorro, el crédito y la inversión mundiales debido a la estabilización de sus economías, a su apertura y liberalización y a las oportunidades masivas de inversión creadas por los programas de privatización. Desde este punto de vista no es inexacto decir que las ciudades latinoamericanas se globalizaron, aunque sí lo sería pensar que asumieron el carácter de ciudades globales. Adicionalmente al papel desempeñado por la expansión financiera y terciaria en la globalización urbana latinoamericana de los noventa habría que considerar el papel de la dimensión ideológica, en donde la arquitectura y la planeación urbana juegan un papel central como vehículos de nuevos "modelos" de ciudad y arquitectura adaptados a las nuevas circunstancias de la economía planetaria. Esta dimensión ayudaría a entender que la velocidad de ciertos procesos de cambio haya podido ser mayor (hipotéticamente, habría que constatarlo), y su estabilidad y consolidación también más grande por el efecto jugado sobre el cambio de mentalidades. La globalización como idea plantea una serie de retos de investigación a ser abordados. En particular es interesante entender cómo un concepto científico como el desarrollado por Sassen asume con el tiempo el carácter de receta de política urbana, se convierte en fórmula mágica de solución de todos los problemas, agenciada tanto por los grandes consultores internacionales (como el caso de Porter en Colombia y probablemente en algunos otros países de América Latina), como por las agencias multilaterales de crédito (BID, BM). Igualmente interesante es una reflexión acerca del papel jugado por la comunidad académica en este sentido: ¿qué tipo de vehículo somos? ¿qué tan conscientes somos de este papel jugado independientemente de nuestra voluntad?

Finalmente, las dimensiones, velocidades y sentido de las transformaciones urbanas constatadas por Sassen deberían ponerse en contraste con lo observado en las ciudades de América Latina y servir de criterio de ponderación, es decir, tener una idea de órdenes de magnitud y velocidades relativas. Esta herramienta es indispensable en ciencias sociales y lamentablemente muy poco utilizada, por lo menos en el medio latinoamericano.

ii) Las escalas

Sassen aborda explícitamente la discusión acerca de las formas de articulación entre las distintas escalas espaciales de funcionamiento económico, especialmente la mundial, nacional y metropolitana (la escala regional subnacional no la aborda explícitamente). A este nivel plantea algunas hipótesis y deriva algunas conclusiones menos sólidas que en los otros campos y fenómenos analizados.

Una de las conclusiones que plantea es la de la más aguda separación entre las economías nacionales y las ciudades globales; como acompañamiento a esta hipótesis entendemos la afirmación hecha de la existencia de las tres ciudades globales como un sistema integrado, con el desempeño de funciones complementarias. Veamos de qué forma expresa cada una de estas afirmaciones y la discusión que suscita. La afirmación acerca de la aguda separación entre las economías nacionales y las ciudades globales la plantea de la siguiente manera. Una de las conclusiones centrales del análisis en el capítulo 6 es: "The evidence suggests there is a pronounced difference between New York, London, and Tokyo, which have extremely high concentrations of producer services and a strong orientation to the global market, and other cities" (p.164). Por tanto, el punto de partida es la evidencia de una diferencia marcada entre estas ciudades globales y otras ciudades de sus redes urbanas nacionales en términos de su orientación al mercado mundial y el peso relativo de los servicios a la producción.

Tomando esta evidencia como punto de partida, plantea una conclusión derivada de la anterior:

The locational distribution of producer services follows, up to a point, the accepted urban hierarchy in each of these countries. (...) While there is, then, a pattern resembling an urban hierarchy, one can also detect severe discontinuities. (...) It would seem that rather than a diffusion of growth along urban hierarchy, there is a clear divergence in growth paths (p.165).

De este razonamiento se extrae entonces una conclusión más general: 
The evidence discussed in this chapter indicates that growth predicated on a global market orientation induces discontinuity in the urban hierarchy. It is perhaps becoming increasingly evident that the centrality of mass production and mass consumption was a crucial factor in creating a balanced urban system and national integration of these countries (p.165).

En el caso de estas evidencias y de las conclusiones derivadas a partir de ellas, el razonamiento de Sassen no es tan cuidadoso como en los casos anteriores y sus debilidades pueden poner en tela de juicio la solidez de las afirmaciones. La primera afirmación recoge la evidencia central del estudio y no tiene lugar a discusión o planteamiento de objeciones. No obstante, ella dice con claridad lo que el estudio obtuvo y las limitaciones de la afirmación: se trabajó solamente el sector de los servicios a la producción y la orientación al mercado mundial como criterios de caracterización de las diferencias entre las ciudades globales y otras ciudades de sus mismas redes urbanas. En ese sentido lo que la evidencia muestra es la especificidad de las ciudades globales en el desempeño de un papel en el nivel de la provisión de este tipo de servicios. No obstante, la segunda afirmación intenta ampliar el ámbito de validez de la primera afirmación al campo más general de las jerarquías urbanas nacionales y de su estructura. Habla entonces ya de una discontinuidad y la asocia a la globalización. Esta pretendida discontinuidad y su asociación con la globalización son del todo discutibles. Primero, porque el estudio no muestra si esta discontinuidad es mayor en esta época que en anteriores y, por tanto, no establece verdaderamente si es un rasgo específico de la fase actual, examen que sí realiza con otras afirmaciones e hipótesis. Más aún, muestra evidencias que lo contradicen pues plantea con claridad que la expansión de los servicios a la producción es más acelerada en las economías nacionales como un todo que en las mismas ciudades globales; adicionalmente, porque los datos de población y empleo agregado en estas ciudades muestran que su participación está descendiendo, es decir no hay polarización económica o laboral hacia ellas. Segundo, porque aún aceptando esta pretendida discontinuidad, no hay ninguna prueba de su asociación con la globalización, ni siquiera con el papel de estas actividades económicas tan específicas. La variedad de elementos a tener en cuenta es muy grande y una afirmación sólida en este sentido ni siquiera es ensayada.

A la discusión de este aspecto específico le atribuyo especial importancia porque ha tenido una particular trascendencia en las formas de representación académica de la globalización. Esta discontinuidad y ruptura de las ciudades globales con sus medios nacionales y sus redes urbanas particularmente, se ha tomado como un hecho establecido y fuera de toda discusión. Además de los defectos lógicos y de fundamento empírico, esta afirmación de la ruptura de escalas se apoya implícitamente en una idea desarrollada en capítulos posteriores que, a nuestro modesto entender, no hace más que poner en evidencia lo contrario, es decir que una parte fundamental de la naturaleza de las ciudades globales deriva de la naturaleza social y económica de los estados y territorios nacionales en los que ella se ubica.

Esta afirmación, corolaria como dijimos, plantea que las tres ciudades globales conforman un sistema en donde no simplemente compiten entre sí sino que se complementan por la influencia que cada una ejerce sobre distintos horarios del mundo y por funciones diferenciales asumidas por cada una:

Furthermore, the book sought to show that in many regards New York, London and Tokyo function as one transterritorial marketplace. These three cities do not simply compete with each other for the same business. They also fulfill distinct roles and function as a triad. Briefly, in the 1980s Tokyo emerged as the main center for the export of capital; London as the main center for the processing of capital, largely through its vast international banking network linking London to most countries in the world and through the Euromarkets; and New York City as the main receiver of capital, the center for investment decisions and for the production of innovations that can maximize profitability. Beyond the often-mentioned need to cover the time zones, there is an operational aspect that suggests a distinct transterritorial economy for a specific set of functions (p.327).

La idea del funcionamiento de las ciudades globales como sistema es pertinente y válida. No obstante, la ilustración de esta idea pone en evidencia el peso mayor de las economías nacionales de cada ciudad en la definición de su rol. Si Tokio opera en los ochentas como exportador, Nueva York como absorbedor y Londres como procesador es porque las condiciones de estas economías nacionales así lo determinaban: es el estado de la economía japonesa, floreciente en los ochenta y aparentemente resistente a las crisis 
experimentadas por los otros países desarrollados, lo que se expresa en el papel de Tokio como exportador de capital; es igualmente la necesidad de financiar los desequilibrios permanentes en la balanza de pagos de los EE.UU. y su insuficiente nivel de ahorro doméstico lo que se refleja en el papel específico de Nueva York como plaza financiera; es probablemente el papel de Londres como articulador del mercado financiero europeo lo que explica su rol peculiar. Si esta lectura se hace 10 años más tarde se verá que estos papeles han cambiado pues Japón viene de una crisis de más de 5 años, los EE.UU. de un alto y estable crecimiento de más de 7 años y Europa de una recuperación menos duradera que la de los EE.UU. pero aparentemente sólida.

iii) Las estructuras de concentración La investigación de Sassen pone en evidencia la naturaleza y los componentes de uno de los elementos de una nueva estructura de concentración surgida como resultado de los cambios operados por la globalización. En particular, muestra en detalle el significado de estos cambios para las llamadas ciudades globales. No obstante, otros componentes de esta estructura no son estudiados $y$, en cierto sentido, son poco valorados por este estudio.

Sassen muestra cómo la decadencia de las ciudades industriales en los países desarrollados se acompañó de un renacimiento urbano en las ciudades globales con base en el desempeño de funciones financieras y terciarias. Muestra cómo otras ciudades industriales no lograron contrabalancear la tendencia decadente y continúan sumidas en crisis severas. No obstante, el componente que no muestra Sassen y que hace falta para una adecuada lectura e interpretación de los procesos, es lo que viene sucediendo con la aparición de nuevos polos manufactureros, con características propias de la época, diferentes de las fases previas de la industrialización. Esta mirada permitiría matizar las afirmaciones de Sassen acerca de la desconexión de las ciudades globales de sus territorios nacionales y probablemente hallar el tipo de interrelaciones que puedan estarse dando entre estos nuevos polos manufactureros y los grandes centros urbanos.

Desde este punto de vista la investigación de Sassen contribuye muy poco a romper con la mirada concentracionista puntual y, más bien, alimenta enfoques puntuales, muy cercanos a las tradicionales discusiones de concentración vs. desconcentración o descentralización. No obstante, es preciso señalar que éste no es el objeto mismo de la investigación de Sassen pero que su tratamiento no tiene el cuidado suficiente para evitar alimentar visiones sesgadas de la problemática socio-espacial. 